\title{
Certainty Equivalence M-MRAC for Systems with Unmatched Uncertainties
}

\author{
Vahram Stepanyan and Kalmanje Krishnakumar
}

\begin{abstract}
The paper presents a certainty equivalence state feedback indirect adaptive control design method for the systems of any relative degree with unmatched uncertainties. The approach is based on the parameter identification (estimation) model, which is completely separated from the control design and is capable of producing parameter estimates as fast as the computing power allows without generating high frequency oscillations. It is shown that the system's input and output tracking errors can be systematically decreased by the proper choice of the design parameters.
\end{abstract}

\section{INTRODUCTION}

Control design for systems with unmatched uncertainties is a challenging task, and the main design method is based on the backstepping technique outlined in [4]. There, it has been shown that certainty equivalence principle leads to over parametrization, which can be avoided by the departure of the certainty equivalence. In this case, the adaptive laws enter into the control law, which can result in the high magnitude control signals in the case of large adaptive rates (fast adaptation). An alternative certainty equivalence control design method is presented in [1], which avoids over parametrization for linear systems, but not for nonlinear systems with the relative degree greater than two.

In this paper, we present a certainty equivalence indirect adaptive control approach without over parametrization for the nonlinear systems of any relative degree, which is the main contribution of the paper. The approach is based on the identification scheme, which is completely separated from the control design. To enable a fast adaptation without generating high frequency oscillations in the adaptive signals, it employs an error feedback term, like in the modified reference model MRAC (M-MRAC) architecture introduced in [5]. It is shown that the state prediction error converges to zero independent of the control design. Moreover, it is shown that transient of the state prediction error and the combined parameter estimation error can be regulated by the proper choice of the error feedback gain and the adaptation rate. The control design follows the command filtered backstepping procedure [2]. It is shown that the input tracking error (difference between ideal control and command filtered certainty equivalence control signal) and output tracking error can be regulated by the proper choice of design parameters.

Vahram Stepanyan is with Mission Critical Technologies Inc. at NASA Ames Research Center, Moffett Field, CA 94035, email: vahram.stepanyan@nasa.gov

Kalmanje Krishnakumar is with Intelligent Systems Division, NASA Ames Research Center, Moffett Field, CA 94035, email: kalmanje.krishnakumar@nasa.gov
The rest of the paper is organized as follows. In Section II, we give the problem statement and the assumptions. In Section III, we introduce the identification model and give its properties. The control design is presented in Section IV, and the controller's performance is analyzed in Section V. A simulation example is presented in Section VI, and some concluding remarks are given in Section VII.

\section{Problem Statement}

Consider an uncertain single input single output (SISO) system in the parametric strict feedback form [4] (p. 99)

$$
\begin{gathered}
\dot{x}_{1}(t)=x_{2}(t)+\boldsymbol{\theta}_{1}^{\top} \boldsymbol{\varphi}_{1}\left(x_{1}\right) \\
\dot{x}_{2}(t)=x_{3}(t)+\boldsymbol{\theta}_{2}^{\top} \boldsymbol{\varphi}_{2}\left(x_{1}, x_{2}\right) \\
\vdots \\
\dot{x}_{n}(t)=u(t)+\boldsymbol{\theta}_{n}^{\top} \boldsymbol{\varphi}_{n}(\boldsymbol{x})
\end{gathered}
$$

with $\boldsymbol{x}(0)=\boldsymbol{x}_{0}$, where $\boldsymbol{x}(t)=\left[x_{1}, \ldots, x_{n}\right]^{\top} \in \mathbb{R}^{n}$ and $u(t) \in \mathbb{R}$ are the state and the input of the system, $\boldsymbol{\theta}_{i} \in \mathbb{R}^{p_{i}}$ are vector of unknown constant parameters, and $\varphi_{i}: \mathbb{R}^{i} \rightarrow$ $\mathbb{R}^{p_{i}}, i=1, \ldots, n$ are sufficiently smooth known vectorfunctions. The system (1) is written in the vector form

$$
\dot{\boldsymbol{x}}(t)=A \boldsymbol{x}(t)+\boldsymbol{b} u(t)+\boldsymbol{\eta}(t),
$$

where we denote

$$
A=\left[\begin{array}{cc}
0_{n-1 \times 1} & \mathbb{I}_{n-1 \times n-1} \\
0 & 0_{1 \times n-1}
\end{array}\right], \boldsymbol{b}=\left[\begin{array}{c}
0_{n-1 \times 1} \\
1
\end{array}\right]
$$

and $\boldsymbol{\eta}(t)=\left[\boldsymbol{\theta}_{1}^{\top} \boldsymbol{\varphi}_{1}\left(x_{1}\right) \boldsymbol{\theta}_{2}^{\top} \boldsymbol{\varphi}_{2}\left(x_{1}, x_{2}\right) \ldots \boldsymbol{\theta}_{n}^{\top} \boldsymbol{\varphi}_{n}(\boldsymbol{x})\right]^{\top}$.

The objective is to design a control signal $u(t)$ such that the closed-loop signals are bounded, and the system's output $y(t)=x_{1}(t)$ tracks the output $y_{r}(t)=x_{r 1}$ of the reference model

$$
\dot{\boldsymbol{x}}_{r}(t)=A_{r} \boldsymbol{x}_{r}(t)+\boldsymbol{b}_{r} r(t), \quad \boldsymbol{x}_{r}(0)=\boldsymbol{x}_{0},
$$

where $A_{r}=A-B \boldsymbol{k}^{\top}, \boldsymbol{b}_{r}=k_{r} \boldsymbol{b}$, and $r(t)$ is a piecewise continuous and bounded external command. Here, the feedback gain $k$ and feedforward gain $k_{r}$ are chosen to make $A_{r}$ Hurwitz and meet the performance specifications.

\section{IDENTIFICATION MODEL}

In order to generate the necessary parameter estimates, we introduce the following identification model

$$
\begin{aligned}
& \dot{\hat{\boldsymbol{x}}}(t)=A \hat{\boldsymbol{x}}(t)+\boldsymbol{b u}(t)+\hat{\boldsymbol{\eta}}(t)+c \tilde{\boldsymbol{x}}(t) \\
& \hat{\boldsymbol{x}}(0)=\hat{\boldsymbol{x}}_{0},
\end{aligned}
$$

where $\hat{\boldsymbol{x}}(t)$ is the state prediction, $\tilde{\boldsymbol{x}}(t)=\boldsymbol{x}(t)-\hat{\boldsymbol{x}}(t)$ is the state prediction error, $c>0$ is a design parameter, 
$\hat{\boldsymbol{\eta}}(t)=\left[\hat{\boldsymbol{\theta}}_{1}^{\top}(t) \boldsymbol{\varphi}_{1}\left(x_{1}\right) \hat{\boldsymbol{\theta}}_{2}^{\top}(t) \boldsymbol{\varphi}_{2}\left(x_{1}, x_{2}\right) \ldots \hat{\boldsymbol{\theta}}_{n}^{\top}(t) \boldsymbol{\varphi}_{n}(\boldsymbol{x})\right]^{\top}$, and $\hat{\boldsymbol{\theta}}_{i}(t)$ is the estimate of the unknown parameter $\boldsymbol{\theta}_{i}$ for each $i=1, \ldots, n$. These estimates are generated according to adaptive laws

$$
\dot{\hat{\boldsymbol{\theta}}}_{i}(t)=\gamma \tilde{x}_{i}(t) \boldsymbol{\varphi}_{i}\left(x_{1}, \ldots, x_{i}\right), \quad i=1, \ldots, n,
$$

where $\gamma>0$ is the adaptation rate. The state prediction error dynamics do not explicitly depend on the control signal

$$
\dot{\tilde{\boldsymbol{x}}}(t)=(A-c \mathbb{I}) \tilde{\boldsymbol{x}}(t)+\tilde{\boldsymbol{\eta}}(t),
$$

where we define $\tilde{\boldsymbol{\eta}}(t)=\boldsymbol{\eta}(t)-\hat{\boldsymbol{\eta}}(t)$ with $\tilde{\boldsymbol{\theta}}_{i}(t)=\boldsymbol{\theta}_{i}-$ $\hat{\boldsymbol{\theta}}_{i}(t), i=1, \ldots, n$ being the parameter estimation error.

Lemma 3.1: The error signals $\tilde{\boldsymbol{x}}(t)$ and $\tilde{\boldsymbol{\theta}}_{i}(t), \quad i=$ $1, \ldots, n$ are globally uniformly bounded, and $\tilde{\boldsymbol{x}}(t) \rightarrow 0$ as $t \rightarrow \infty$.

Proof: Consider a candidate Lyapunov function

$$
V(t)=\frac{1}{2} \sum_{i=1}^{n}\left[\tilde{x}_{i}^{2}(t)+\frac{1}{\gamma} \tilde{\boldsymbol{\theta}}_{i}^{\top}(t) \tilde{\boldsymbol{\theta}}_{i}(t)\right]
$$

the derivative of which is computed along the trajectories of the prediction error dynamics (6) and the adaptive laws (5)

$$
\begin{gathered}
\dot{V}(t)=\tilde{\boldsymbol{x}}^{\top}(t)(A-c \mathbb{I}) \tilde{\boldsymbol{x}}(t) \\
+\sum_{i=1}^{n} \tilde{\boldsymbol{\theta}}_{i}^{\top}(t)\left[\tilde{x}_{i}(t) \boldsymbol{\varphi}_{i}\left(x_{1}, \ldots, x_{i}\right)+\frac{\dot{\tilde{\boldsymbol{\theta}}}_{i}(t)}{\gamma}\right] .
\end{gathered}
$$

Substituting the adaptive laws and completing the squares results in

$$
\begin{aligned}
& \dot{V}(t)=-(c-1) \sum_{i=1}^{n} \tilde{x}_{i}^{2}(t)-\frac{1}{2}\left[\tilde{x}_{1}^{2}(t)+\tilde{x}_{n}^{2}(t)\right] \\
& -\frac{1}{2} \sum_{i=1}^{n-1}\left[\tilde{x}_{i}(t)-\tilde{x}_{i+1}(t)\right]^{2} \leq-(c-1) \sum_{i=1}^{n} \tilde{x}_{i}^{2}(t) .
\end{aligned}
$$

With $c>1$, the LaSalle-Yoshizawa theorem ( [4], p.24) guarantees that $\tilde{\boldsymbol{x}}(t), \tilde{\boldsymbol{\theta}}_{i}(t), i=1, \ldots, n$ are globally uniformly bounded, and $\tilde{\boldsymbol{x}}(t) \rightarrow 0$ as $t \rightarrow \infty$. In particular, there exists $\beta_{1}>0$ such that $\sum_{i=1}^{n}\left\|\tilde{\boldsymbol{\theta}}_{i}(t)\right\|^{2} \leq \beta_{1}^{2}$.

Lemma 3.2: If $\hat{\boldsymbol{x}}_{0}=\boldsymbol{x}_{0}$, then the state prediction error $\tilde{\boldsymbol{x}}(t)$ satisfies the bound

$$
\|\tilde{\boldsymbol{x}}(t)\|_{\mathcal{L}_{\infty}} \leq \beta_{1} \gamma^{-1 / 2} .
$$

Proof: The proof follows from the fact that $\|\tilde{\boldsymbol{x}}(t)\|^{2} \leq$ $2 V(t) \leq 2 V(0) \leq \beta_{1}^{2}$ for all $t \geq 0$.

The next lemma gives the bound on the state prediction error when $\hat{\boldsymbol{x}}_{0} \neq \boldsymbol{x}_{0}$.

Lemma 3.3: If $\hat{\boldsymbol{x}}_{0} \neq \boldsymbol{x}_{0}$, then $\tilde{\boldsymbol{x}}(t)$ satisfies the bound

$$
\|\tilde{\boldsymbol{x}}(t)\| \leq \beta_{2} e^{-(c-1) t}+\frac{\beta_{1}}{\sqrt{\gamma}},
$$

where $\beta_{2}=\sqrt{\left|2 V(0)-\frac{\beta_{1}^{2}}{\gamma}\right|}$.

Proof: The inequality (8) can be written as

$$
\dot{V}(t) \leq-2(c-1) V(t)+\frac{c-1}{\gamma} \beta_{1}^{2},
$$

which implies that

$$
V(t) \leq\left[V(0)-\frac{\beta_{1}^{2}}{2 \gamma}\right] e^{-2(c-1) t}+\frac{\beta_{1}^{2}}{2 \gamma} \leq c_{3} e^{-2 k t}+\frac{c}{\gamma} .
$$

Recalling that $\|\tilde{\boldsymbol{x}}(t)\|^{2} \leq 2 V(t)$, we obtain

$$
\|\tilde{\boldsymbol{x}}(t)\| \leq \sqrt{\left[2 V(0)-\frac{\beta_{1}^{2}}{\gamma}\right] e^{-2(c-1) t}+\frac{\beta_{1}^{2}}{\gamma}},
$$

The bound (10) follows from the fact that $\sqrt{a+b} \leq \sqrt{a}+\sqrt{b}$ for any $a \geq 0, b \geq 0$.

Since the effect of the initialization of the state prediction error decays exponentially with the rate $c-1$, and $c$ will be assigned to large values in order to damp the high frequency oscillations in adaptive estimates for large adaptation rates, we set $\hat{\boldsymbol{x}}_{0}=\boldsymbol{x}_{0}$ in the following derivations.

Next lemma gives the bounds on the error signal $\tilde{\boldsymbol{\eta}}(t)$ and a tighter bound on $\tilde{\boldsymbol{x}}(t)$, when $\boldsymbol{x}(t)$ and $u(t)$ are bounded (which will be provided with the control design in the next section).

Lemma 3.4: Let the estimates $\hat{\boldsymbol{x}}(t)$ and $\hat{\boldsymbol{\eta}}(t)$ be generated by the identification model (4) and (5). In addition, let $\boldsymbol{x}(t)$ and $u(t)$ be bounded. Then $\tilde{\boldsymbol{\eta}}(t)$ and $\tilde{\boldsymbol{x}}(t)$ satisfy the following bounds

$$
\begin{array}{r}
\|\tilde{\boldsymbol{\eta}}(t)\| \leq \beta_{2} e^{-\nu_{1} t}+\frac{\beta_{3}}{\sqrt{\gamma}} \\
\|\tilde{\boldsymbol{x}}(t)\| \leq \beta_{4} e^{-\nu_{2} t}+\frac{\beta_{5}}{c \sqrt{\gamma}},
\end{array}
$$

where the constants $\beta_{i}>0, i=2, \ldots, 5$ and $\nu_{1}>\nu_{2}>0$ are defined in the proof.

Proof: It is straightforward to show that $\tilde{\eta}_{i}(t)$ satisfies the dynamics

$$
\begin{gathered}
\ddot{\tilde{\eta}}_{i}(t)+c \dot{\tilde{\eta}}_{i}(t)+\gamma \rho_{i}(t) \tilde{\eta}_{i}(t)=-\gamma \rho_{i}(t) \tilde{x}_{i+1}(t) \\
-\gamma \dot{\rho}_{i}(t) \tilde{x}_{i}(t)+c h_{i}(t)+\dot{h}_{i}(t)
\end{gathered}
$$

for all $i=1, \ldots, n$, where for the notational convenience we introduce a variable $\tilde{x}_{n+1}=0$. The other variables are defined as $\rho_{i}(t)=\boldsymbol{\varphi}_{i}^{\top}(t) \boldsymbol{\varphi}_{i}(t), h_{i}(t)=\tilde{\boldsymbol{\theta}}_{i}^{\top}(t) \dot{\boldsymbol{\varphi}}_{i}(t)$. Since $\boldsymbol{x}(t)$ and $u(t)$ are bounded, it follows that $\dot{\boldsymbol{x}}(t)$ is bounded. Therefore, there exist positive constants $\delta_{1}, \delta_{2}, \delta_{3}$ such that $\left\|\rho_{i}(t)\right\|_{\mathcal{L}_{\infty}} \leq \delta_{1},\left\|\dot{\rho}_{i}(t)\right\|_{\mathcal{L}_{\infty}} \leq \delta_{2}$ and $\left\|h_{i}(t)\right\|_{\mathcal{L}_{\infty}} \leq$ $\delta_{3}$. Then, it can be concluded from equation (16) that $c$ determines the damping and $\gamma$ determines the frequency of the signal $\tilde{\eta}_{i}(t)$. It follows from the results of [5], that choosing $c \geq 2 \sqrt{\delta_{1} \gamma}$ damps the oscillations in $\tilde{\boldsymbol{\eta}}(t)$ and guarantees the bound

$$
\|\tilde{\boldsymbol{\eta}}(t)\| \leq \beta_{2} e^{-\nu_{2} t}+\delta_{4}\|\tilde{\boldsymbol{x}}(t)\|+\frac{\delta_{5}}{\sqrt{\gamma}}\|\boldsymbol{h}(t)\|,
$$

where $\nu_{2}$ is proportional to $\sqrt{\gamma}$, and the positive constants $\beta_{2}, \delta_{4}$ and $\delta_{5}$ are independent of $\gamma$ (see details in [5]). Substituting (9) we arrive to (14) with $\beta_{3}=\delta_{4} \beta_{1}+\delta_{3} \delta_{5}$.

Since $A_{r}$ is Hurwitz, there exist positive constants $\delta_{6}$ and $\nu_{2}$ such that the state transition matrix satisfies the inequality $\left\|e^{A t}\right\| \leq \delta_{6} e^{-\nu_{2} t}$. It follows that $\left\|e^{(A-c \mathbb{I}) t}\right\| \leq \delta_{6} e^{-\left(\nu_{2}+c\right) t}$. Then we obtain from (6) by the direct integration that

$$
\begin{gathered}
\|\tilde{\boldsymbol{x}}(t)\| \leq \frac{\beta_{2} \delta_{6}}{\nu_{2}+c-\nu_{1}}\left[e^{-\nu_{1} t}-e^{-\left(\nu_{2}+c\right) t}\right] \\
+\frac{\beta_{3}}{\sqrt{\gamma}} \frac{\delta_{6}}{\nu_{2}+c}\left[1-e^{-\left(\nu_{2}+c\right) t}\right] \leq \beta_{4} e^{-\nu_{2} t}+\frac{\beta_{5}}{c \sqrt{\gamma}},
\end{gathered}
$$

since $c$ and $\nu_{1}$ are proportional to $\sqrt{\gamma}$, which is much greater than $\nu_{2}$ (adaptation is faster than the reference model). 


\section{Control Design}

In this section, we design four controllers, first three of which are used for the analysis purposes, and only the last one is implemented.

\section{A. Known System}

For the analysis purposes we first design a controller assuming that $\boldsymbol{\theta}$ is known (ideal control). Following the standard backstepping procedure [4], we define new variables

$$
z_{i}^{0}(t)=x_{i}^{0}(t)-\alpha_{i-1}^{0}(t), \quad i=1, \ldots, n
$$

(superscript 0 indicates that the variables corresponds to the ideal control signal) and stabilizing functions

$$
\begin{gathered}
\alpha_{0}^{0}(t)=0, \quad \alpha_{1}^{0}(t)=-\eta_{1}(t) \\
\alpha_{i}^{0}(t)=-\eta_{i}(t)+\dot{\alpha}_{i-1}^{0}(t), \quad i=2, \ldots, n .
\end{gathered}
$$

The system (2) can be written in new variables as

$$
\dot{\boldsymbol{z}}^{0}(t)=A \boldsymbol{z}^{0}(t)+\boldsymbol{b}\left[u^{0}(t)-\alpha_{n}^{0}(t)\right]
$$

Obviously, the control signal

$$
u^{0}(t)=-\boldsymbol{k}^{\top} \boldsymbol{z}^{0}(t)+k_{r} r(t)+\alpha_{n}^{0}(t),
$$

reduces the system into the reference model (3), hence the error $\boldsymbol{e}^{0}(t)=\boldsymbol{z}^{0}(t)-\boldsymbol{x}_{r}(t)$ satisfies dynamics

$$
\dot{e}^{0}(t)=A_{r} \boldsymbol{e}^{0}(t)
$$

Lemma 4.1: The controller defined by (19), (20) and (22) guarantees the control objective for the system (2).

Proof: Since $A_{r}$ is Hurwitz, it follows that $e^{0}(t) \in \mathcal{L}_{\infty}$ and $e^{0}(t)$ exponentially converges to zero. Hence, $y^{0}(t)=$ $y_{r}(t)+e_{1}^{0}(t)$ exponentially converges to $y_{r}(t)$. In addition, from $r(t) \in \mathcal{L}_{\infty}$ it follows that $\boldsymbol{x}_{r}(t) \in \mathcal{L}_{\infty}$, which along with $\boldsymbol{e}^{0}(t) \in \mathcal{L}_{\infty}$ implies that $\boldsymbol{z}^{0}(t) \in \mathcal{L}_{\infty}$. Boundedness of $\alpha_{i}^{0}(t), i=1, \ldots, n$ and $\boldsymbol{x}^{0}(t)$ is obtained recursively starting with $x_{1}^{0}(t)=z_{1}^{0}(t) \in \mathcal{L}_{\infty}$. Then, $u^{0}(t) \in \mathcal{L}_{\infty}$ follows.

\section{B. Certainty Equivalent Control}

Next, we design a controller for the identification model (4), by replacing the unknown parameter $\boldsymbol{\theta}$ with its estimate $\hat{\boldsymbol{\theta}}(t)$ in the stabilizing functions

$$
\begin{gathered}
\hat{\alpha}_{0}(t)=0, \quad \hat{\alpha}_{1}(t)=-\hat{\eta}_{1}(t) \\
\hat{\alpha}_{i}(t)=-\hat{\eta}_{i}(t)+\dot{\hat{\alpha}}_{i-1}(t), \quad i=2, \ldots, n,
\end{gathered}
$$

and introducing new variables as

$$
\hat{z}_{i}(t)=\hat{x}_{i}(t)-\hat{\alpha}_{i-1}(t), \quad i=1, \ldots, n .
$$

The identification model in new variables takes the form

$$
\dot{\hat{\boldsymbol{z}}}(t)=A \hat{\boldsymbol{z}}(t)+\boldsymbol{b}\left[u(t)-\hat{\alpha}_{n}(t)\right]+c \tilde{\boldsymbol{x}}(t) .
$$

Defining the control signal as

$$
\hat{u}(t)=-\boldsymbol{k}^{\top} \hat{\boldsymbol{z}}(t)+k_{r} r(t)+\hat{\alpha}_{n}(t) .
$$

we obtain

$$
\dot{\hat{\boldsymbol{z}}}(t)=A_{r} \hat{\boldsymbol{z}}(t)+\boldsymbol{b}_{r} r(t)+c \tilde{\boldsymbol{x}}(t),
$$

which is in the form of modified reference model introduced in [5]. In this case the error signal $\hat{\boldsymbol{e}}(t)=\hat{\boldsymbol{z}}(t)-\boldsymbol{x}_{r}(t)$ evolves according to the dynamics

$$
\dot{\hat{\boldsymbol{e}}}(t)=A_{r} \hat{\boldsymbol{e}}(t)+c \tilde{\boldsymbol{x}}(t) .
$$

Lemma 4.2: The controller defined by (24), (25) and (27) guarantees the control objective.

Proof: According to Lemma 3.1, $\tilde{\boldsymbol{x}}(t) \in \mathcal{L}_{\infty}$ and $\tilde{\boldsymbol{x}}(t) \rightarrow 0$ as $t \rightarrow \infty$. Since $A_{r}$ is Hurwitz and $r(t) \in \mathcal{L}_{\infty}$, it follows that $\hat{\boldsymbol{z}}(t) \in \mathcal{L}_{\infty}, \hat{\boldsymbol{e}}(t) \in \mathcal{L}_{\infty}$ and $\hat{\boldsymbol{e}}(t) \rightarrow 0$. Therefore, application of the controller defined by (24), (25) and (27) to both the system and the identification model results in

$$
y(t)=y_{r}(t)+\hat{e}_{1}(t)+\tilde{x}_{1}(t) \rightarrow y_{r}(t)
$$

In addition, from $y_{r}(t) \in \mathcal{L}_{\infty}$ it follows that $x_{1}(t) \in \mathcal{L}_{\infty}$, hence $\hat{\alpha}_{1}(t) \in \mathcal{L}_{\infty}$, since $\boldsymbol{\varphi}_{1}\left(x_{1}\right)$ is continuous and $\hat{\boldsymbol{\theta}}_{1}(t) \in$ $\mathcal{L}_{\infty}$ according to Lemma 3.1. This implies that $\hat{x}_{2}(t) \in \mathcal{L}_{\infty}$ and $x_{2}(t) \in \mathcal{L}_{\infty}$. Continuing this recursion we conclude that $\hat{\boldsymbol{x}}(t) \in \mathcal{L}_{\infty}, \boldsymbol{x}(t) \in \mathcal{L}_{\infty}, \hat{\alpha}_{i}(t) \in \mathcal{L}_{\infty}, i=1, \ldots, n$, $\dot{\hat{\alpha}}_{i}(t) \in \mathcal{L}_{\infty}, i=1, \ldots, n-1$, and $\hat{u}(t) \in \mathcal{L}_{\infty}$.

We notice that using the state $\boldsymbol{x}$ in the identification model (4) simplifies the stability analysis in the identification stage. However, the control design becomes problematic, because $\dot{\hat{\alpha}}_{i}(t)$ contains unknown parameter $\boldsymbol{\theta}$ through the state derivative $\dot{\boldsymbol{x}}(t)$. One way to overcome this issue is to replace $\varphi_{i}\left(x_{1}, \ldots, x_{i}\right)$ with $\varphi_{i}\left(\hat{x}_{1}, \ldots, \hat{x}_{i}\right)$ in the identification model (4), which brings additional terms $\lambda_{i}\left\|\boldsymbol{\theta}_{i}\right\| \tilde{\boldsymbol{x}}^{\top}(t) \tilde{\boldsymbol{x}}(t)$ into the $\dot{V}(t)$ expression (8), where $\lambda_{i}$ is the Lipschitz constant of $\varphi_{i}\left(x_{1}, \ldots, x_{i}\right)$. In this case the same stability properties are guaranteed with the choice of $c>1+$ $\sum_{i=1}^{n} \lambda_{i}\left\|\boldsymbol{\theta}_{i}\right\|$, and $\dot{\hat{\alpha}}_{i}(t)$ becomes implementable. However, the repetitive differentiations of $\hat{\boldsymbol{\theta}}_{i}(t)$ introduces the multiple powers of the adaptation rate $\gamma$ into the control law, which makes the designer to keep the adaptation slow from the perspective of the control constraints. This is in conflict with the improvement of the performance by speeding up the adaptation. From this perspective, we use the command filtered backstepping approach from [2]. Although the method was introduce to simplify the process of determining the command derivatives in the backstepping procedure, it also allows to completely separate the identifier design from the controller design. Therefore, the identification process can be made as fast as the computational power allows. In the meantime, the high frequency oscillations associated with the fast adaptation are avoided with the proposed identification model by the proper choice of design parameters.

\section{Command Filtering}

Following [2], we introduce the command filtered approach for the design (19), (20) and (22), which will be used for the analysis purposes, and for the design (24), (25) and (27), which will actually be implemented. In the case of known system, the new variables are introduced as (superscript $f$ indicates that the command filtered version)

$$
z_{i}^{f}(t)=x_{i}^{f}(t)-\sigma_{i-1,1}^{0}(t), \quad i=1, \ldots, n,
$$


where the command filter is designed as

$$
\begin{gathered}
\dot{\sigma}_{i, 1}^{0}(t)=\omega \sigma_{1,2}^{0}(t) \\
\dot{\sigma}_{i, 2}^{0}(t)=-2 \zeta \omega \sigma_{i, 2}^{0}(t)-\omega\left[\sigma_{i, 1}^{0}(t)-\alpha_{i}^{f}(t)\right] \\
i=0, \ldots, n-1,
\end{gathered}
$$

with the initial conditions $\sigma_{i, 1}^{0}(0)=\alpha_{i}^{f}(0)$ and $\sigma_{i, 2}^{0}(0)=0$, and the stabilizing functions are defined as

$$
\begin{gathered}
\alpha_{0}^{f}(t)=0, \quad \alpha_{1}^{f}(t)=-\eta_{1}^{f}(t) \\
\alpha_{i}^{f}(t)=-\eta_{i}^{f}(t)+\omega \sigma_{i-1,2}^{0}(t), \quad i=2, \ldots, n,
\end{gathered}
$$

where the superscript $f$ indicates that the corresponding quantities are computed when the command filtered control is in the loop. The system (2) is written in $z$-variable as

$$
\dot{\boldsymbol{z}}^{f}(t)=A \boldsymbol{z}^{f}(t)+\boldsymbol{b}\left[u(t)-\alpha_{n}^{f}(t)\right]+\overline{\boldsymbol{\alpha}}^{f}(t)-\overline{\boldsymbol{\sigma}}^{0}(t),
$$

where

$$
\overline{\boldsymbol{\alpha}}^{f}(t)=\left[\begin{array}{c}
\alpha_{1}^{f}(t) \\
\vdots \\
\alpha_{n-1}^{f}(t) \\
0
\end{array}\right], \overline{\boldsymbol{\sigma}}^{0}(t)=\left[\begin{array}{c}
\sigma_{1,1}^{0}(t) \\
\vdots \\
\sigma_{n-1,1}^{0}(t) \\
0
\end{array}\right]
$$

The compensated state is introduced as $\boldsymbol{v}^{0}(t)=\boldsymbol{z}^{f}(t)-$ $\boldsymbol{\xi}^{0}(t)$, where $\boldsymbol{\xi}^{0}(t)$ is defined dynamically as

$$
\dot{\xi}_{i}^{0}(t)=\sigma_{i, 1}^{0}(t)-\alpha_{i}^{f}(t)+\xi_{i+1}^{0}(t)
$$

with $\xi_{i}^{0}(0)=0$ for $i=1, \ldots, n-1$, and $\xi_{n}^{0}(t)=0$. In compensated state the system (2) takes the form

$$
\dot{\boldsymbol{v}}^{0}(t)=A \boldsymbol{v}^{0}(t)+\boldsymbol{b}\left[u(t)-\alpha_{n}^{f}(t)\right],
$$

The control signal $u(t)$ in this procedure is defined as

$$
u^{f}(t)=-\boldsymbol{k}^{\top} \boldsymbol{v}^{0}(t)+k_{r} r(t)+\alpha_{n}^{f}(t) .
$$

Whereas the compensated error signal $\boldsymbol{e}_{c}^{0}(t)=\boldsymbol{v}^{0}(t)-\boldsymbol{x}_{r}(t)$ satisfies the dynamics

$$
\dot{e}_{c}^{0}(t)=A_{r} e_{c}^{0}(t)
$$

and obviously is exponentially stable, the uncompensated error $\boldsymbol{e}^{f}(t)=\boldsymbol{z}^{f}(t)-\boldsymbol{x}_{r}(t)$ has dynamics

$$
\dot{\boldsymbol{e}}^{f}(t)=A_{r} \boldsymbol{e}^{f}(t)-\overline{\boldsymbol{\alpha}}^{f}(t)+\overline{\boldsymbol{\sigma}}^{0}(t) .
$$

Lemma 4.3: The command filtered controller defined by (31), (32), (33), (35) and (37) guarantees the following relationships

$$
\begin{array}{rlrl}
e^{f}(t)-e^{0}(t) & =\mathcal{O}(\varepsilon), & \boldsymbol{\xi}^{0}(t)=\mathcal{O}(\varepsilon) \\
\sigma_{i, 1}^{0}(t)-\alpha_{i}^{0}(t) & =\mathcal{O}(\varepsilon), & & i=1, \ldots, n-1 \\
\omega \sigma_{i, 2}^{0}(t)-\dot{\alpha}_{i}^{0}(t) & =\mathcal{O}(\varepsilon), & & i=1, \ldots, n-1,
\end{array}
$$

where $\varepsilon=1 / \omega$ (the proper choice of $\zeta$ and $\omega$ is discussed in [2]), and the notation $\mathcal{O}(\varepsilon)$ is adopted from [3] (p. 383).

Proof: Although the error systems (38) and (39) are not in the standard backstepping form, the proof still follows the steps from [2].

Now we design the last controller, which is actually implemented. It is the command filtered version of the certainty equivalent control, designed in the previous subsection. The uncompensated state is now introduced as

$$
z_{i}(t)=\hat{x}_{i}(t)-\sigma_{i-1,1}(t), \quad i=1, \ldots, n .
$$

where $\sigma_{i-1,1}(t)$ is the filter's state given by

$$
\begin{gathered}
\dot{\sigma}_{i, 1}(t)=\omega \sigma_{1,2}(t) \\
\dot{\sigma}_{i, 2}(t)=-2 \zeta \omega \sigma_{i, 2}(t)-\omega\left[\sigma_{i, 1}(t)-\hat{\alpha}_{i}^{f}(t)\right] \\
i=0, \ldots, n-1,
\end{gathered}
$$

with the initial conditions $\sigma_{i, 1}(0)=\hat{\alpha}_{i}^{f}(0)$ and $\sigma_{i, 2}(0)=0$, and the stabilizing functions have the form

$$
\begin{gathered}
\hat{\alpha}_{0}^{f}(t)=0, \quad \hat{\alpha}_{1}^{f}(t)=-\hat{\eta}_{1}^{f}(t) \\
\hat{\alpha}_{i}^{f}(t)=-\hat{\eta}_{i}^{f}(t)+\sigma_{i-1,2}(t), \quad i=2, \ldots, n .
\end{gathered}
$$

Here, we we introduce a short hand notation $\hat{\eta}_{i}^{f}(t)=$ $\hat{\boldsymbol{\theta}}_{i}^{\top}(t) \boldsymbol{\varphi}^{f}(t)$ and $\boldsymbol{\varphi}_{i}^{f}(t)=\boldsymbol{\varphi}\left(x_{1}^{f}(t), \ldots, x_{i}^{f}(t)\right)$. The identification model in $z$-variables takes the form

$$
\begin{gathered}
\dot{\boldsymbol{z}}(t)=A \boldsymbol{z}(t)+\boldsymbol{b}\left[u(t)-\hat{\alpha}_{n}^{f}(t)\right] \\
+c \tilde{\boldsymbol{x}}(t)+\overline{\hat{\boldsymbol{\alpha}}}^{f}(t)-\overline{\boldsymbol{\sigma}}(t) .
\end{gathered}
$$

The compensated state and its dynamics for the identification model are similarly introduced

$$
\dot{\xi}_{i}(t)=\sigma_{i, 1}(t)-\hat{\alpha}_{i}^{f}(t)+\xi_{i+1}(t)
$$

with $\xi_{i}(0)=0$ for $i=1, \ldots, n-1, \xi_{n}(t)=0$, and $\boldsymbol{v}(t)=$ $\boldsymbol{z}(t)-\boldsymbol{\xi}(t)$,

$$
\dot{\boldsymbol{v}}(t)=A \boldsymbol{v}(t)+\boldsymbol{b}\left[u(t)-\hat{\alpha}_{n}^{f}(t)\right]+c \tilde{\boldsymbol{x}}(t) .
$$

The control signal to be implemented has the form

$$
u(t)=-\boldsymbol{k}^{\top} \boldsymbol{v}(t)+k_{r} r(t)+\hat{\alpha}_{n}^{f}(t) .
$$

The resulting compensated error signal $\boldsymbol{e}_{c}(t)=\boldsymbol{v}(t)-\boldsymbol{x}_{r}(t)$ and uncompensated error signal $\boldsymbol{e}(t)=\boldsymbol{z}(t)-\boldsymbol{x}_{r}(t)$ satisfy dynamics

$$
\begin{gathered}
\dot{\boldsymbol{e}}_{c}(t)=A_{r} \boldsymbol{e}_{c}(t)+c \tilde{\boldsymbol{x}}(t) \\
\dot{\boldsymbol{e}}(t)=A_{r} \boldsymbol{e}(t)-\overline{\hat{\boldsymbol{\alpha}}}(t)+\overline{\boldsymbol{\sigma}}(t)+c \tilde{\boldsymbol{x}}(t) .
\end{gathered}
$$

Lemma 4.4: The command filtered controller defined by (41), (42), (43), (45) and (47) guarantees the following relationships

$$
\begin{aligned}
\boldsymbol{e}(t)-\hat{\boldsymbol{e}}(t) & =\mathcal{O}(\varepsilon), & & \boldsymbol{\xi}(t)=\mathcal{O}(\varepsilon) \\
\sigma_{i, 1}(t)-\hat{\alpha}_{i}(t) & =\mathcal{O}(\varepsilon), & & i=1, \ldots, n-1 \\
\omega \sigma_{i, 2}(t)-\dot{\hat{\alpha}}_{i}(t) & =\mathcal{O}(\varepsilon), & & i=1, \ldots, n-1 .
\end{aligned}
$$

Proof: Since the exponential convergence of $\tilde{\boldsymbol{x}}(t)$ is not guaranteed, Tikhonov's theorem ( [3], Theorem 11.2) cannot be directly applied to the system comprised of (44), (45) and (49). However, since $\tilde{\boldsymbol{x}}(t)$ does not depend on $\varepsilon$, a simple state transformation $\boldsymbol{s}(t)=\boldsymbol{e}(t)-\boldsymbol{\mu}(t)$, where $\boldsymbol{\mu}(t)$ is dynamically defined as $\dot{\boldsymbol{\mu}}(t)=A_{r} \boldsymbol{\mu}(t)+c \tilde{\boldsymbol{x}}(t)$, results in the system $\dot{\boldsymbol{s}}(t)=A_{r} \boldsymbol{s}(t)-\overline{\hat{\boldsymbol{\alpha}}}(t)+\overline{\boldsymbol{\sigma}}(t)$, for which the hypothesis of the Thikhonov's theorem can be verified following the steps from [2], and the last two relationships in (50) can be concluded along with $\boldsymbol{s}(t)-\hat{\boldsymbol{s}}(t)=\mathcal{O}(\varepsilon)$, where $\hat{\boldsymbol{s}}(t)=$ $\hat{\boldsymbol{e}}(t)-\boldsymbol{\mu}(t)$ and satisfies the exponentially stable dynamics $\dot{\hat{\boldsymbol{s}}}(t)=A_{r} \hat{\boldsymbol{s}}(t)$. It follows that $\boldsymbol{e}(t)-\hat{\boldsymbol{e}}(t)=\boldsymbol{s}(t)-\hat{\boldsymbol{s}}(t)=$ $\mathcal{O}(\varepsilon)$, which completes the proof. 


\section{Performance Analysis}

The following two lemmas are needed to prove our main result.

Lemma 5.1: Let the command filtered controller for system (2) be defined by (31), (32), (33), (35) and (37). Then all closed-loop signals are bounded and

$$
\boldsymbol{x}^{f}(t)-\boldsymbol{x}^{0}(t)=\mathcal{O}(\varepsilon) .
$$

In addition, if $\omega$ is sufficiently large, then

$$
u^{f}(t)-u^{0}(t)=\mathcal{O}(\varepsilon) .
$$

Proof: Since $e^{0}(t) \in \mathcal{L}_{\infty}$ it follows from (40) that $\boldsymbol{e}^{f}(t) \in \mathcal{L}_{\infty}$, implying that $\boldsymbol{z}^{f}(t) \in \mathcal{L}_{\infty}$. It follows form Lemma 4.1 and Lemma 4.3 that $\sigma_{i, 1}^{0}(t) \in \mathcal{L}_{\infty}$ and $\sigma_{i, 2}^{0}(t) \in$ $\mathcal{L}_{\infty}$ for $i=1, \ldots, n-1$. Then, (31) implies that $\boldsymbol{x}^{f}(t) \in$ $\mathcal{L}_{\infty}$. Therefore $\boldsymbol{\eta}^{f}(t) \in \mathcal{L}_{\infty}$, hence $\alpha_{i}^{f}(t) \in \mathcal{L}_{\infty}$ for all $i=1, \ldots, n$. Since $\boldsymbol{e}_{c}(t)$ is exponentially stable, it follows that $\boldsymbol{v}^{0}(t) \in \mathcal{L}_{\infty}$, and hence $u^{f}(t) \in \mathcal{L}_{\infty}$.

It is straightforward to compute the difference

$$
x_{i}^{f}(t)-x_{i}^{0}(t)=e_{i}^{f}(t)-e_{i}^{0}(t)+\sigma_{i, 1}^{0}(t)-\alpha_{i}^{0}(t) .
$$

Since $e_{i}^{f}(t)-e_{i}^{0}(t)=\mathcal{O}(\varepsilon)$ and $\sigma_{i, 1}^{0}(t)-\alpha_{i}^{0}(t)=\mathcal{O}(\varepsilon)$, it follows that $x_{i}^{f}(t)-x_{i}^{0}(t)=\mathcal{O}(\varepsilon)$ for all $i=1, \ldots, n$. Next, we compute the difference

$$
u^{f}(t)-u^{0}(t)=-\boldsymbol{k}^{\top}\left[\boldsymbol{v}^{0}(t)-\boldsymbol{z}^{0}(t)\right]+\alpha_{n}^{f}(t)-\alpha_{n}^{0}(t) .
$$

Since $\boldsymbol{\xi}^{0}(t)=\mathcal{O}(\varepsilon)$, it follows that $\boldsymbol{v}^{0}(t)-\boldsymbol{z}^{0}(t)=\mathcal{O}(\varepsilon)$. On the other hand $\alpha_{n}^{f}(t)-\alpha_{n}^{0}(t)=\eta_{n}^{f}(t)-\eta_{n}(t)+$ $\omega \sigma_{n-1,2}^{0}(t)-\dot{\alpha}_{n-1}^{0}(t)$. Since $\boldsymbol{\varphi}_{n}(\boldsymbol{x})$ is smooth, we have $\boldsymbol{\varphi}_{n}\left(\boldsymbol{x}^{f}(t)\right)-\boldsymbol{\varphi}_{n}\left(\boldsymbol{x}^{0}(t)\right)=\mathcal{O}(\varepsilon)$. Then it follows that $u^{f}(t)-u^{0}(t)=\mathcal{O}(\varepsilon)$ if $\omega>\max \left(\|\boldsymbol{k}\|,\left\|\boldsymbol{\theta}_{n}\right\|\right)$.

Lemma 5.2: Let the command filtered controller for system (2) and identification model (4) be defined by (31), (32), (33), (35) and (37). Then all signals are bounded and

$$
\hat{\boldsymbol{x}}^{f}(t)-\hat{\boldsymbol{x}}(t)=\mathcal{O}(\varepsilon) .
$$

In addition, if $\omega$ is sufficiently large, then

$$
\hat{u}^{f}(t)-\hat{u}(t)=\mathcal{O}(\varepsilon) .
$$

The lemma is proved similar to the previous one.

Theorem 5.1: Let the system's controller be defined according to command filtered scheme given by (41), (42), (43), (45) and (47). Then the input and output tracking errors satisfy the following upper bounds

$$
\begin{aligned}
& |\tilde{u}(t)| \leq \beta_{6} e^{-\nu_{1} t}+\frac{\beta_{7}}{\sqrt{\gamma}}+\mathcal{O}(\varepsilon) \\
& |e(t)| \leq \beta_{8} e^{-\nu_{2} t}+\frac{\beta_{9}}{\sqrt{\gamma}}+\mathcal{O}(\varepsilon),
\end{aligned}
$$

where $\tilde{u}(t)=\left\|u^{0}(t)-u(t)\right\|, \beta_{6}, \beta_{7}, \beta_{8}, \beta_{9}$ and $\nu$ are positive constants defined in the proof.

Proof: It is easy to see that

$$
\begin{aligned}
\tilde{u}(t) & =u(t)-u^{f}(t)+u^{f}(t)-\hat{u}^{f}(t) \\
& =\mathcal{O}(\varepsilon)-\boldsymbol{k}^{\top} \tilde{\boldsymbol{v}}(t)+\tilde{\alpha}_{n}^{f}(t),
\end{aligned}
$$

where $\tilde{\boldsymbol{v}}(t)=\boldsymbol{v}^{0}(t)-\boldsymbol{v}(t)$ and $\tilde{\alpha}_{n}(t)=\alpha_{n}^{f}(t)-\hat{\alpha}_{n}^{f}(t)$. Obviously, $\tilde{\boldsymbol{v}}(t)$ satisfies the dynamics

$$
\dot{\tilde{\boldsymbol{v}}}(t)=A_{r} \tilde{\boldsymbol{v}}(t)+c \tilde{\boldsymbol{x}}(t)
$$

with the initial conditions $\tilde{\boldsymbol{v}}(0)=\tilde{x}(0)-\tilde{\boldsymbol{\eta}}(0)$, where we denote $\tilde{\eta}_{i}(t)=\eta_{i}^{f}(t)-\hat{\eta}_{i}^{f}(t)=\tilde{\boldsymbol{\theta}}_{i}(t) \boldsymbol{\varphi}_{i}^{f}(t)$ for each $i=$ $1, \ldots, n$. similar to (18), one can obtain form (58) that

$$
\|\tilde{\boldsymbol{v}}(t)\| \leq \beta_{10} e^{-\nu_{2} t}+\frac{\beta_{11}}{\sqrt{\gamma}}
$$
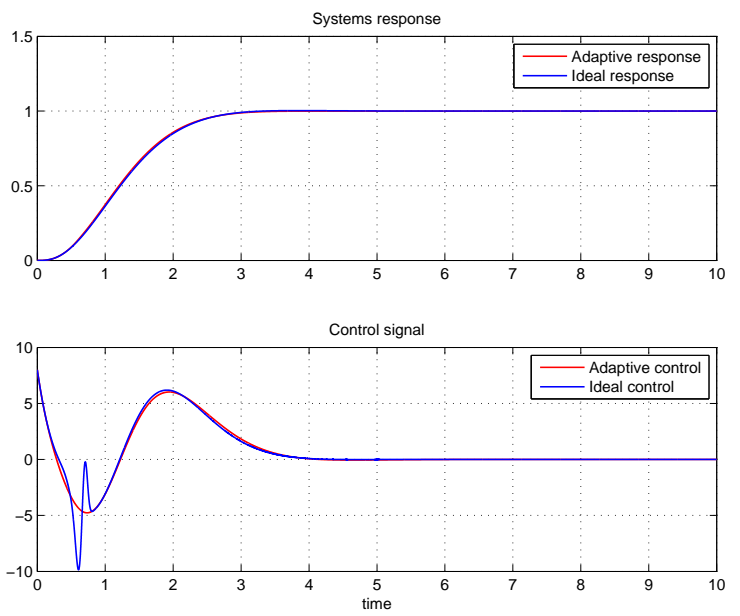

Fig. 1. Adaptive step response and corresponding adaptive control signal vs ideal step response and ideal control signal.

Next, we observe that the signals $\tilde{\sigma}_{i, 1}(t)=\sigma_{i, 1}^{0}(t)-\sigma_{i, 1}(t)$ and $\tilde{\sigma}_{i, 2}(t)=\sigma_{i, 2}^{0}(t)-\sigma_{i, 2}(t)$ satisfy the operator equations

$$
\begin{array}{cc}
\tilde{\sigma}_{i, 1}(s)=G_{1}(s) \tilde{\alpha}_{i}^{f}(s), & G_{1}(s)=\frac{\omega^{2}}{s^{2}+2 \zeta \omega s+\omega^{2}} \\
\tilde{\sigma}_{i, 2}(s)=G_{2}(s) \tilde{\alpha}_{i}^{f}(s), & G_{2}(s)=\frac{\omega s}{s^{2}+2 \zeta \omega s+\omega^{2}},
\end{array}
$$

where $\tilde{\alpha}_{i}^{f}(t)=\alpha_{i}^{f}(t)-\hat{\alpha}_{i}^{f}(t)$. Since $\left\|G_{1}(s)\right\|_{\mathcal{H}_{\infty}}=1$ for $\zeta \geq \sqrt{2} / 2$ and $\left\|G_{2}(s)\right\|_{\mathcal{H}_{\infty}}=(2 \zeta)^{-1}$, it follows from [3] (p. 201) that

$$
\begin{gathered}
\left\|\tilde{\sigma}_{i, 1}(t)\right\|_{\mathcal{L}_{\infty}} \leq\left\|\tilde{\alpha}_{i}^{f}(t)\right\|_{\mathcal{L}_{\infty}} \\
\left\|\tilde{\sigma}_{i, 2}(t)\right\|_{\mathcal{L}_{\infty}} \leq(2 \zeta)^{-1}\left\|\tilde{\alpha}_{i}^{f}(t)\right\|_{\mathcal{L}_{\infty}} .
\end{gathered}
$$

Now we can recursively compute the bound on $\tilde{\alpha}_{n}^{f}(t)$ using the definitions (33) and (43). For $i=1$, we have $\tilde{\alpha}_{1}^{f}(t)=\tilde{\eta}_{1}^{f}(t)$, therefore

$$
\left|\tilde{\alpha}_{1}(t)\right| \leq \beta_{2} e^{-\nu_{1} t}+\frac{\beta_{3}}{\sqrt{\gamma}} .
$$

For $i=2, \ldots, n$, we have $\tilde{\alpha}_{i}^{f}(t)=-\tilde{\eta}_{i}(t)+\omega \tilde{\sigma}_{i, 2}(t)$, hence

$$
\left|\tilde{\alpha}_{n}(t)\right| \leq q_{n} \beta_{2} e^{-\nu_{1} t}+\frac{q_{n} \beta_{3}}{\sqrt{\gamma}},
$$

where $q_{n}=1+\frac{\omega}{2 \zeta}+\cdots+\left(\frac{\omega}{2 \zeta}\right)^{n-1}$.

Combining the relationships (57), (59) and (63) we obtain (55), where $\beta_{6}=\|\boldsymbol{k}\| \beta_{10}+q_{n} \beta_{2}, \beta_{7}=\|\boldsymbol{k}\| \beta_{11}+q_{n} \beta_{3}$, since $\nu_{1}>\nu_{2}$ for large values of $\gamma$ (fast adaptation).

To prove (56), we notice that $y(t)-y_{r}(t)=\tilde{x}_{1}(t)+$ $\hat{x}_{1}^{f}(t)-x_{r 1}(t)=\tilde{x}_{1}^{f}(t)+e_{1}(t)$. Since $e_{1}(t)=\hat{e}_{1}(t)+\mathcal{O}(\varepsilon)$, it follows that $y(t)-y_{r}(t)=\tilde{x}_{1}(t)+\hat{e}_{1}(t)+O(\varepsilon)$. Using (18) one can obtain from (29) that

$$
\|\hat{\boldsymbol{e}}(t)\| \leq \beta_{12} e^{-\nu_{2} t}+\frac{\beta_{13}}{\sqrt{\gamma}},
$$


and the relationship (56) follows. The proof is complete.

It follows form Theorem 5.1 that the bounds on the input and output tracking errors can be systematically decreased by choosing large values for $\omega$ and $\gamma$.
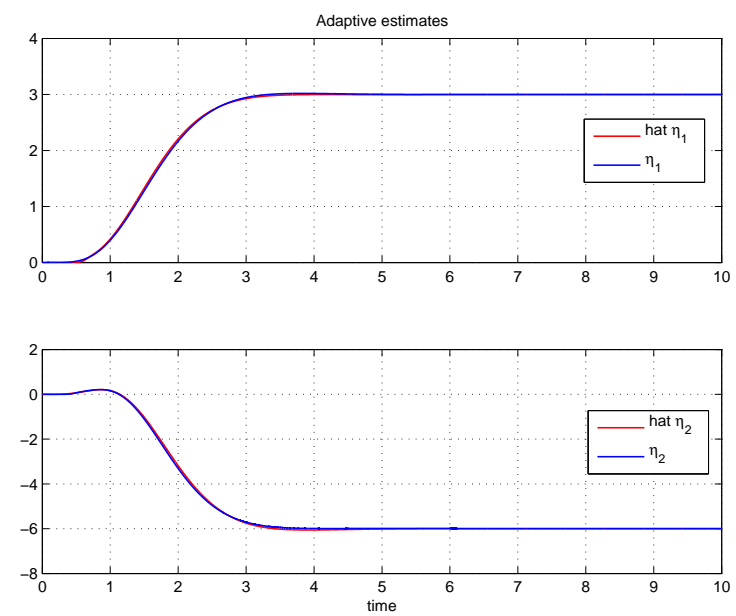

Fig. 2. Estimates of $\eta_{1}$ and $\eta_{2}$ vs true values for the step input.
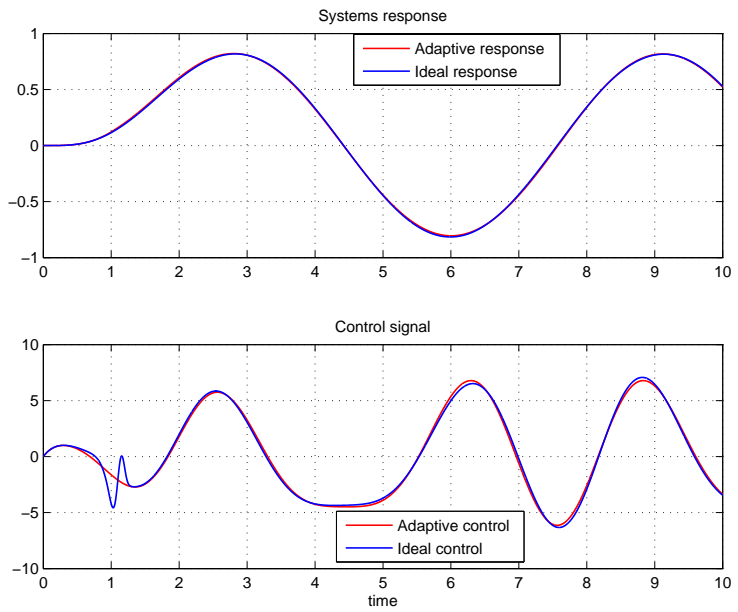

Fig. 3. Adaptive sinusoidal response and corresponding adaptive control signal vs ideal step response and ideal control signal.

Remark 5.1: The proposed approach can be readily extended to multi-input-multi-output systems with unmatched uncertainties. Also, time variant parameters and external disturbances can be introduced in the proposed approach.

\section{Simulation Results}

As a simulation example we consider a third order system

$$
\begin{gathered}
\dot{x}_{1}(t)=x_{2}(t)+\theta_{1} x_{1}^{2}(t) \\
\left.\dot{x}_{2}(t)=x_{3}(t)+\theta_{2} x_{1}(t) x_{2}(t)\right) \\
\dot{x}_{3}(t)=u(t)
\end{gathered}
$$

with the unknown parameters $\theta_{1}=3$ and $\theta_{2}=2$. The reference model is selected with $\boldsymbol{k}^{\top}=\left[\begin{array}{lll}8 & 10.4 & 5.2\end{array}\right]$ and $k_{r}=8$. The identification model is designed with $c=495$ and $\gamma=500000$. For the command filtering we set $\omega=500$ and $\zeta=0.8$. Figure 1 displays systems response to step input along with the command filtered certainty equivalent control signal vs the systems step response (ideal response) and control signal (ideal control) for the conventional backstepping design assuming parameters are known. The identifier's performance is displayed in Figure 2. It can be observed that good tracking is achieved in all signals for the unit step command. The proposed controller achieves a similar performance for the sinusoidal command as can be seen from Figures 3 and 4 .
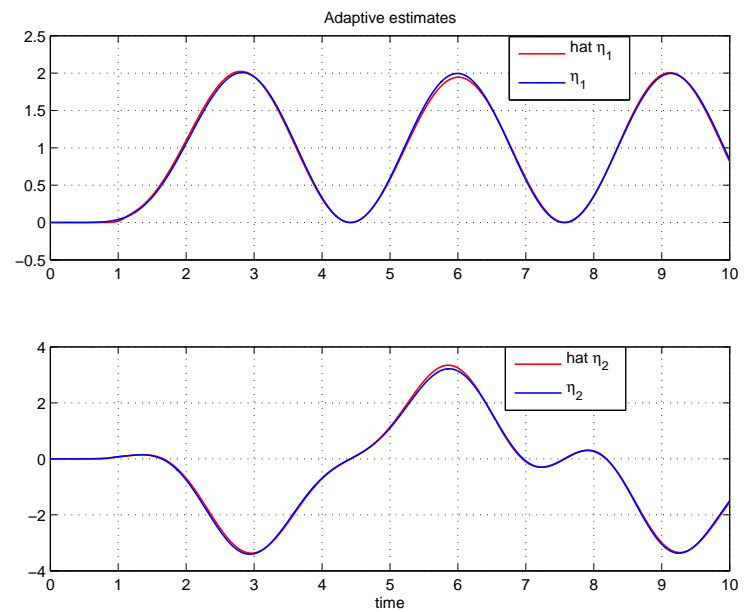

Fig. 4. Estimates of $\eta_{1}$ and $\eta_{2}$ vs true values for the sinusoidal input.

\section{CONCLUDING REMARKS}

We have presented an indirect adaptive control method for nonlinear systems with unmatched uncertainties that follows the certainty equivalence principle. The approach uses a fast identification model, which is independent of the control design and achieves desired transient and steady state properties by the proper choice of the design parameters. The controller is in the form of the command filtered backstepping control. The resulting tracking errors can be decreased as desired by speeding up the adaptation and command filtering processes, subject to available computational power.

\section{REFERENCES}

[1] J. D. Boskovic and Z. Han, "Certainty Equivalence Adaptive Control of Plants With Unmatched Uncertainty Using State Feedback," IEEE Transactions on Automatic Control, vol. 54, no. 8, pp. 1918 - 1924, 2009.

[2] J. A. Farrell, M. Polycarpou, M. Sharma, and W. Dong, "Command Filtered Backstepping." IEEE Transactions on Automatic Control, vol. 54, no. 6, pp. 1391-1395, June 2009.

[3] H. Khalil, Nonlinear Systems, Third Edition. Prentice Hall, New Jersey, 2002.

[4] M. Krstic, I. Kanellakopoulos, and P. Kokotovic, Nonlinear and Adaptive Control Design. John Wiley \& Sons, New York, 1995.

[5] V. Stepanyan and K. Krishnakumar, "M-MRAC for Nonlinear Systems with Bounded Disturbances," In Proc. of the IEEE Conference on Decision and Control, Orlando, FL, 2011. 